\title{
Influence of Stokes Number on Collisional Interfacial Area Production Terms within the $\Sigma-Y$ Eulerian Spray Atomization Model
}

\author{
Dominik Eichler*, Philipp Pischke, Reinhold Kneer \\ Institute of Heat and Mass Transfer, RWTH Aachen University \\ *Corresponding author: eichler@wsa.rwth-aachen.de
}

\begin{abstract}
The present study shows the effects of Stokes number on the modeling of collisional interfacial area production terms within the $\Sigma-Y$ model. This model can be employed for CFD simulations of high Weber and Reynolds number sprays using a RANS turbulence modeling. Within the model production of interfacial area is assumed to result from turbulent stretching and turbulent droplet collisions. The modeling of collisional processes requires the calculation of a characteristic turbulent collision velocity. In the present work this velocity was determined under consideration of Stokes number effects leading to turbulent droplet velocity fluctuations attenuated with respect to the gas phase fluctuations and including partial correlation between the velocities. The influence of this new modeling approach is tested within a 2D spray simulation by comparing the Sauter mean diameters observed to the ones obtained by employing the modeling approaches proposed in the literature which do not consider any Stokes number effects. The reduced collision velocites in the new modeling lead to higher values for Sauter mean diameters in the spray.
\end{abstract}

\section{Keywords}

CFD, eulerian spray modeling, stochastic collision modeling

\section{Introduction}

High Weber and Reynolds number sprays, as to be found in Diesel fuel injection or rocket fuel injection, can be modeled by the $\Sigma-Y$ Eulerian spray atomization model, originally proposed by Vallet et al. [1]. Here, a transport equation for the liquid mass fraction $Y$ (instead of a transport equation for liquid volume fraction) is employed and another transport equation for $\Sigma$ describing the evolution of volumetric interfacial area (dimension $1 / \mathrm{m}$ ). Liquid and gas are assumed to have identical velocities for the bulk flow. Thus, only one set of momentum equations is sufficient to describe the two-phase flow. Mixing of liquid and gas is performed by turbulent diffusion only. Considering sprays with high Weber and Reynolds numbers, the large scales are assumed to be independent of small scales. Thus, the momentum equations do not feature any effects of surface tension nor laminar viscosity. In the $\Sigma$ - $Y$ model, source and sink terms within the $\Sigma$-equation are assumed to arise from turbulent fluctuations of the flow, which lead to turbulent breakup, turbulent droplet collisions leading to fragmentation or coalescence, or turbulent stretching. Employing a RANS eddy viscosity model, as proposed as one alternative by various authors, see for example [1, 2, 3], the Favre averaged transport equations for liquid mass, momentum and interfacial area can be stated as follows:

$$
\begin{aligned}
& \frac{\partial \bar{\rho} \widetilde{Y}}{\partial t}+\operatorname{div}(\bar{\rho} \widetilde{Y} \widetilde{\mathbf{u}})=\operatorname{div}\left(\bar{\rho} \frac{\nu_{\mathrm{t}}}{\operatorname{Sc}_{\mathrm{t}}} \operatorname{grad}(\widetilde{Y})\right) \\
& \frac{\partial \bar{\rho} \widetilde{u}_{i}}{\partial t}+\operatorname{div}\left(\bar{\rho} \widetilde{u}_{i} \widetilde{\mathbf{u}}\right)=\operatorname{div}\left(\bar{\rho} \nu_{\mathrm{t}} \operatorname{grad}\left(\widetilde{u}_{i}\right)\right) \\
& \frac{\partial \bar{\Sigma}}{\partial t}+\operatorname{div}(\bar{\Sigma} \widetilde{\mathbf{u}})=\operatorname{div}\left(\frac{\nu_{\mathrm{t}}}{\operatorname{Sc}_{\mathrm{t}}} \operatorname{grad}(\bar{\Sigma})\right)+\dot{\Sigma}_{\mathrm{P}}-\dot{\Sigma}_{\mathrm{D}}
\end{aligned}
$$

Here, $\mathrm{Sc}_{\mathrm{t}}$ is the turbulent Schmidt number, and $\dot{\Sigma}_{\mathrm{P}}$ and $\dot{\Sigma}_{\mathrm{D}}$ describe the production and destruction terms for the $\Sigma$ equation, respectively. According to Vallet et al. [1], production and destruction of $\Sigma$ are comprised of two mechanisms:

$$
\begin{aligned}
& \dot{\Sigma}_{\mathrm{P}, \mathrm{TS}}=A \Sigma \\
& \dot{\Sigma}_{\mathrm{P}, \text { Coll }}=\frac{\Sigma}{\tau_{\text {coll }}}\left(1-\frac{\Sigma}{\Sigma_{\text {eq }}}\right)
\end{aligned}
$$

The first mechanism, see equation (4), describes the turbulent stretching of the interface. $A$ is a production rate, proportional to the local velocity gradient. The second one, see equation (5), represents the combined production and destruction of interfacial area by droplet collisions. Here, $\tau_{\text {coll }}$ is a characteristic collisional production time, depending on the relative velocity and spacing between droplets, and $\Sigma_{\text {eq }}$ is the equilibrium value for interfacial area. The mathematical form in equation (5) has been adopted in several publications of other authors, see for example [2, 3, 4, 5, 6, 7, 8]. However, different formulations for the modeling of $\tau_{\text {coll }}$ and $\Sigma_{\text {eq }}$ exist.

Within the present study, a discussion about the modeling of the collisional time $\tau_{\text {coll }}$ and equilibrium value $\Sigma_{\text {eq }}$ for the application in the $\Sigma$ - $Y$ Eulerian model is given, concerning the velocity scale to be chosen. 


\section{Modeling of collisional time scales and equilibrium values: state of the art}

\section{Characteristic collision time}

Vallet et al. propose in [1] the following value:

$$
\tau_{\text {coll, Vallet }}=C_{\tau, \text { Vallet }} \frac{n^{-\frac{1}{3}}}{\Delta u} \text {. }
$$

In equation (6), $n$ is the local number density of droplets. It can be derived under the assumption that the interfacial area is composed of identical droplets. Their correspdonding Sauter mean diameter can be obtained in the following way:

$$
d_{32}=\frac{6 \bar{\rho} Y}{\rho_{\text {liq }} \Sigma}
$$

For further details, see [1]. $n^{-\frac{1}{3}}$ is the local mean distance of two droplets. $\Delta u$ is a characteristic collision velocity, defined as

$$
\Delta u=\left(\varepsilon n^{-\frac{1}{3}}\right)^{\frac{1}{3}} .
$$

The collision velocity is thus represented by the gas phase velocity fluctuations of a turbulent eddy of size $n^{-\frac{1}{3}}$. $C_{\tau, \text { Vallet }}$ is a constant. Vallet et al. propose in [1] a value of $C_{\tau, \text { Vallet }}=1.0 \ldots 1.35$, whereas Demoulin et al. [6] use a value of $C_{\tau, \text { vallet }}=0.3$, see [6].

Beau et al. 5 propose another formulation for the characteristic collisional time inspired by stochastic droplet collision modeling. The collision velocity is set to the rms value of turbulent gas phase fluctuations:

$$
\tau_{\text {coll, Beau }}=\frac{1}{\sqrt{\Delta u} \Sigma} \text { and } \Delta u=\sqrt{\frac{2}{3} k} .
$$

\section{Equilbrium value of interfacial area}

Vallet et al. [1] derive an expression for an equilibrium diameter by evaluation of an energy balance of two identical colliding droplets. It is assumed that all kinetic energy with respect to the center of mass of the system of colliding droplets is converted into surface energy after collision. Thus, an equilibrium diameter can be obtained, which can be transformed into an equilibrium value of interfacial area by equation (7). This finally leads to

$$
d_{\text {eq }}=C_{\text {eq }, \text { Vallet }} \frac{2 \sigma^{\frac{3}{5}} l_{\mathrm{t}}^{\frac{2}{5}}}{k^{\frac{3}{5}}} \frac{(\bar{\rho} Y)^{\frac{2}{15}}}{\rho_{\text {liq }}^{\frac{11}{15}}}
$$

According to [1], the value of the constant is $C_{\text {eq, Vallet }}=1.2$. In [6] one can find $C_{\text {eq, Vallet }}=1.0$ and in $[3]$ a value of $C_{\text {eq, }}$ vallet $=2.5$ is chosen.

Beau et al. [5] propose another formulation for the equilibrium value of interfacial area. They assume that an equilibrium diameter can be determined according to an equilibrium collisional Weber number, defined as

$$
\mathrm{We}_{\mathrm{eq}}=\frac{\rho_{\mathrm{liq}} \Delta u^{2} d_{32}}{\sigma} \text { and } \Delta u^{2}=\frac{2}{3} k
$$

A value for the equilibrium Weber number is not proposed in [5], but Lebas et al. [2] propose a value of $\mathrm{We}_{\mathrm{eq}}=12$ using the same modeling approach.

A confirmation that a drop size distribution undergoing turbulent collisions tends to an equilibrium Weber number was given in $[9]$.

\section{Modeling of collisional time scale and equilibrium value: present approach}

Within the modeling approaches presented above, effects of particle inertia are not considered, which may have an influence on the turbulent fluctutations of the droplet phase and the relative velocity between droplets: Particles dispersed in a turbulent flow react to turbulent velocity fluctuations of the surrounding fluid on different time scales, depending on their turbulent Stokes number. The latter is defined as the ratio of particle relaxation time and a turbulent time scale. Under assumption of an exponential spatial or temporal correlation function for the fluid velocity, Levins and Glastonbury (temporal) [10] and Williams and Crane (spatial) [11] could derive an expression for the mean square turbulent velocity fluctuation of component $i$ of a particle depending on the corresponding velocity fluctuation of the surrounding fluid and the particle's turbulent Stokes number:

$$
\frac{\overline{u_{\mathrm{p}, i}^{2}}}{\overline{\overline{u_{\mathrm{f}, i}^{2}}}}=\frac{1}{1+\mathrm{St}_{\mathrm{t}}}
$$

with

$$
\mathrm{St}_{\mathrm{t}}=\frac{\tau_{\mathrm{p}}}{T_{\mathrm{t}}} \text { and } \tau_{\mathrm{p}}=\frac{\rho_{\mathrm{p}} d_{\mathrm{p}}^{2}}{18 \mu} \text { (assuming Stokes drag) }
$$


Since the particles will be influenced mainly by the most energetic large turbulent structures, $T_{\mathrm{t}}$ is chosen as the integral turbulent time scale. Considering equations 12 and 13 , small particles tend to follow the turbulent fluctuations ideally, whereas large particles are not affected by turbulent fluctuations. The collisional velocity needed is the relative velocity between droplets, calculated as the absolute value of the velocity difference vectors of two droplets:

$$
\begin{aligned}
\overline{\Delta u_{\mathrm{p}}^{2}} & =\sum_{i=1}^{3} \overline{\left(u_{\mathrm{p}, 1, i}-u_{\mathrm{p}, 2, i}\right)^{2}} \\
& =\sum_{i=1}^{3}[\overbrace{\overline{u_{\mathrm{p}, 1, i}^{2}}+\overline{u_{\mathrm{p}, 2, i}^{2}}}^{\text {uncorrel. }}-2 \overbrace{\overline{u_{\mathrm{p}, 1, i} u_{\mathrm{p}, 2, i}}}^{\text {correl. }}]
\end{aligned}
$$

Thus, the velocity difference between two particles consists of an uncorrelated and a correlated part. Taking into account the definition of turbulent kinetic energy, the uncorrelated part can be specified as

$$
\left(\overline{\Delta u_{\mathrm{p}}^{2}}\right)_{\text {uncorrel. }}=\frac{4 k}{\left(1+\mathrm{St}_{\mathrm{t}, 1}\right)\left(1+\mathrm{St}_{\mathrm{t}, 2}\right)} .
$$

The correlation term is evaluated for a high density ratio between particle and gas phase and the application of a suitable form for the covariance of the fluid velocity surrounding the particle, see [11]. Williams and Crane finally calculate the following universal expression for the ratio of the mean square of particle relative velocity to the mean square of fluid velocity fluctuations, applicable to the whole Stokes number range:

$$
\frac{\overline{\Delta u_{\mathrm{p}, i}^{2}}}{\overline{u_{\mathrm{f}, i}^{2}}}=\frac{\left(\mathrm{St}_{1}+\mathrm{St}_{2}\right)^{2}-4 \mathrm{St}_{1} \mathrm{St}_{2}\left[\frac{1+\mathrm{St}_{1}+\mathrm{St}_{2}}{\left(1+\mathrm{St}_{1}\right)\left(1+\mathrm{St}_{2}\right)}\right]^{0.5}}{\left(\mathrm{St}_{1}+\mathrm{St}_{2}\right)\left(1+\mathrm{St}_{1}\right)\left(1+\mathrm{St}_{2}\right)}
$$

A droplet distribution is not known within the $\Sigma-Y$ model, but a local Sauter mean diameter. Thus, the turbulent Stokes numbers are set to be equal for both droplets. Furthermore, individual droplet velociy components are not known and isotropic turbulence is assumed leading to the following absolute value for the mean square of particle relative velocity:

$$
\overline{\Delta u_{\mathrm{p}}^{2}}=\frac{2 \mathrm{St}}{1+\mathrm{St}^{2}}\left(1-\frac{(1+2 \mathrm{St})^{0.5}}{1+\mathrm{St}}\right) \cdot 2 k
$$

In Figure 1 a graphical representation of the mathematical relationship between Stokes number and particle velocity fluctuation for the case of identical droplets is shown representing equation (17). It can be seen that small Stokes numbers lead to well correlated velocities between droplets since they simply adjust to the gas phase fluctuations. With increasing Stokes numbers the velocity correlation diminishes leading to higher relative velocites between droplets. A maximum value of $\overline{\Delta u_{\mathrm{p}}^{2}} / 2 k \approx 0.13$ is reached at about $\mathrm{St}=3.5$. For higher values of the Stokes number the influence of turbulent gas phase fluctuations on the motion of particles decreases, which reduces the velocity fluctuations of the droplets and hence the relative velocity between them.

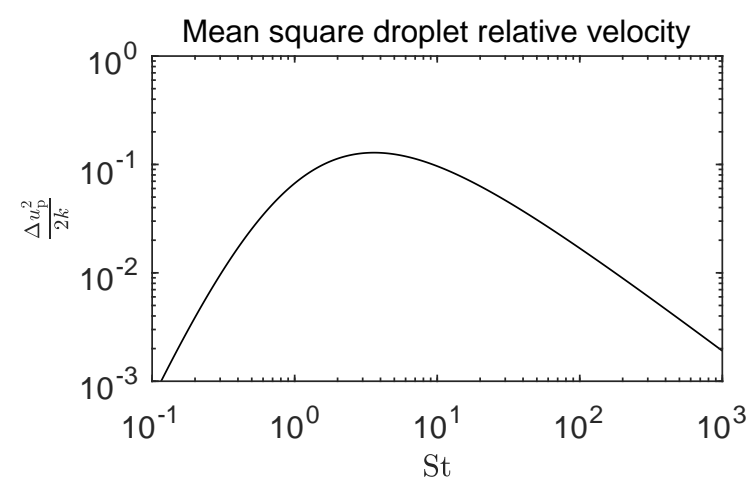

Figure 1. Dependency of particle relative velocity on turbulent Stokes number for identical droplets [11]

Using the stochastic modeling approach for the turbulent collision process within $\Sigma-Y$ model, as proposed in [5], the characteristic collisional time can finally be stated as

$$
\tau_{\text {coll, present }}=\frac{1}{\sqrt{\overline{\Delta u_{\mathrm{p}}^{2}}} \Sigma} .
$$


The equlibrium value for interfacial area is modeled by the definition of an equilibrium collisional Weber number, similar to [5]. But in the new approach presented here the velocity is set according to equation [18):

$$
\mathrm{We}_{\text {eq, present }}=\frac{\rho_{\mathrm{p}} \overline{\Delta u_{\mathrm{p}}^{2}} d_{32}}{\sigma}
$$

In the following sections of this paper the results of CFD simulations for the different modeling approaches introduced above are presented. Attention is given to the influence of the velocity scale on the resulting droplet diameters.

\section{Set-up of the CFD spray simulations}

The $\mathrm{C}_{++}$library OpenFOAM v2.3.0 is used to solve the discretized partial differential equations describing the governing equations for the spray. The dimensions of the computational domain are adopted from [3]. Both, liquid fuel and gas are modeled as incrompressible fluids since the investigation of effects of compressibility are not part of this study. Evaporation is not considered. A $k-\varepsilon$ turbulence model is used with standard values for all constants. Simulations of spray injections for the three different modeling approaches of Vallet et al. [1], Beau et al. [5] and the present one including Stokes number effects are performed. The constants for the collisional production rate and equilibrium value within Vallet et al.'s modeling are set to $C_{\tau, \text { Vallet }}=0.3$ and $C_{\text {eq,Vallet }}=2.5$, respectively. These values are similar to the ones used in [3]. Concerning the transport equation for interfacial area $\Sigma$ only the collisional interfacial area production terms are investigated. All other production terms are switched off. The liquid is injected with a homogeneous velocity (block profile) of $v_{\text {inj }}=350 \mathrm{~m} / \mathrm{s}$ over 6 cells, which corresponds to a nozzle diameter of $d_{\text {nozzle }}=112 \mu \mathrm{m}$.

\section{Results and discussion}

The results obtained for the different modeling approaches of collisional surface production terms are presented. In all figures, the data shown correspond to a time of $t=1000 \mu$ s after start of injection.
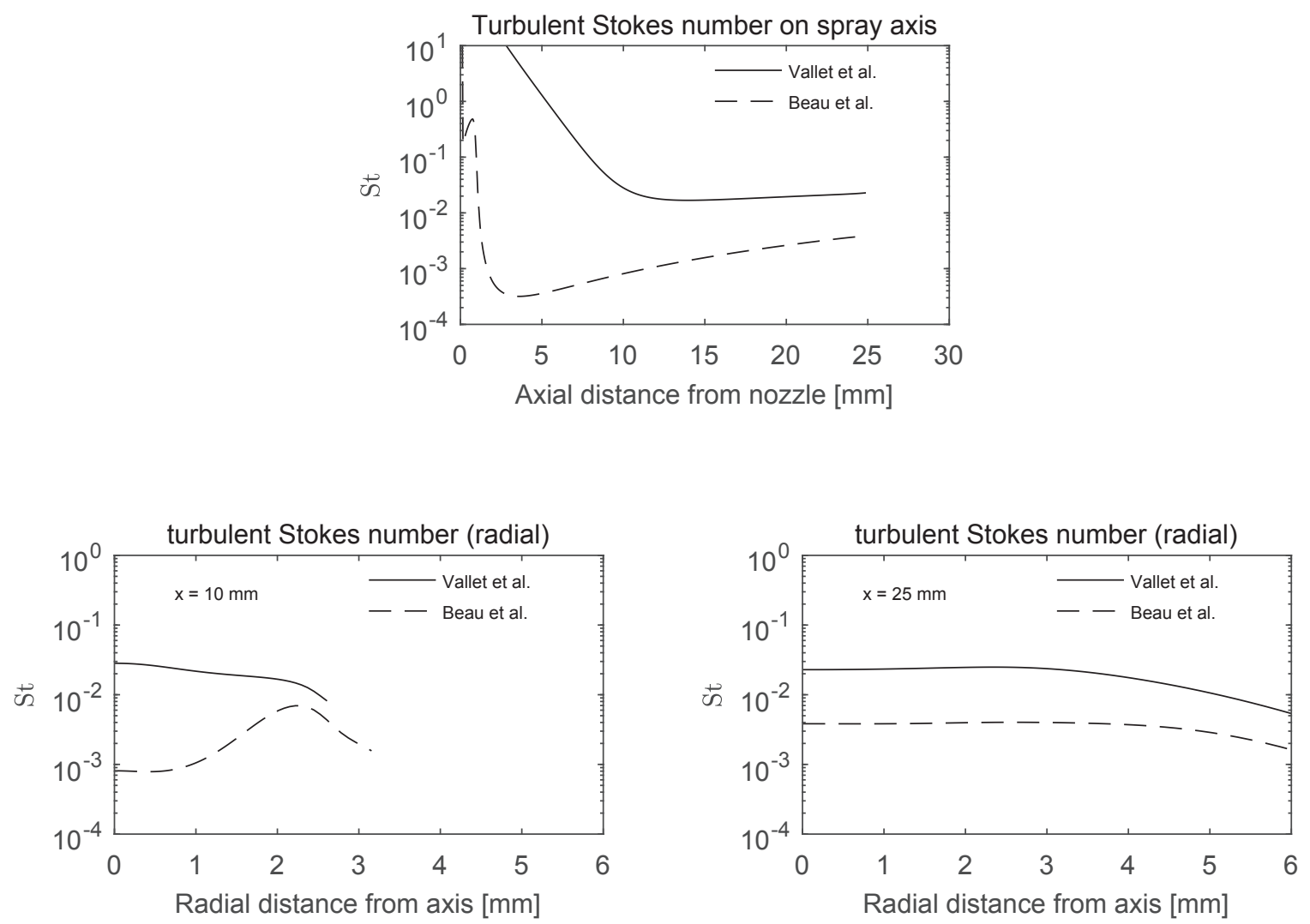

Figure 2. Results of CFD spray simulation showing the axial (top) and radial (bottom) distribution of turbulent Stokes numbers if the collisional surface production terms proposed in Vallet et al. [1] and Beau et al. [5] are used.

\section{Stokes number distribution within the spray}

Taking into account Stokes number effects for the modeling of collisional surface production terms within $\Sigma-Y$ model leads to a modification of the corresponding collisional velocity scale. For example, the maximum possible ratio of the particle relative velocity used in the present modeling and the one proposed by Beau et al. [5] is $\Delta u_{\mathrm{p}, \text { present }} / \Delta u_{\mathrm{p}, \text { Beau }} \approx 0.63$. 
In order to show the range of Stokes numbers present in a spray, the distribution of turbulent Stokes number along the spray axis and the radial distribution of Stokes number at two different axial positions are presented in Figure 2 The modeling approaches of Vallet et al. and Beau et al. are applied, which do not consider any Stokes number effects. The simulations show that Stokes numbers in most of the spray reach from St $\approx 3 \cdot 10^{-4} \ldots 2 \cdot 10^{-2}$. Hence, the consideration of Stokes number effects would lead to significantly decreased collisional velocities, see Figure 1 or equation 18. The impact of these effects on the Sauter mean diameter distribution within a spray is investigated in the following paragraph.

\section{Evolution of Sauter mean diameter}

The reduction of collisional relative velocities between particles, if Stokes number effects are considered (new approach presented here), leads to increased collisional time scales, see equation (19), and increased equilibrium droplet diameters, see equation [20, in comparison to the modeling approach by Beau et al. [5]. This behavior can be observed in Figure 3 which presents the evolution of Sauter mean diameter along the spray axis (left) and the radial distribution of Sauter mean diameter at an axial distance of $x=25 \mathrm{~mm}$ from the nozzle (right) for the three different modeling approaches. The results show that at $x=0 \mathrm{~mm}$ the Sauter mean diameter for all modeling approaches is identical $\left(d_{32} \approx 60 \mu \mathrm{m}\right)$ which satisfies the boundary value set for $\Sigma$ at the nozzle exit. The modeling of Beau et al. [5] features the lowest values for $d_{32}$ concerning both the axial and radial distribution. Regarding the values on the spray axis it can be observed that after an initial shrinking of $d_{32}$ to a value of about $d_{32} \approx 0.2 \mu \mathrm{m}$ near the nozzle, Sauter mean diameters are increasing to values of about $d_{32} \approx 3 \mu \mathrm{m}$. Qualitatively, the same trend is obtained for the present modeling approach but at significantly higher values of $d_{32}$ : After a shrinking to $d_{32} \approx 4 \mu \mathrm{m}$, droplet diameters increase to $d_{32} \approx 40 \mu \mathrm{m}$. The evolution of Sauter mean diameter observed on the axis for both modeling approaches is a result of decreasing values of turbulent kinetic energy with increasing axial distance from the nozzle. Collisional velocities used in both modeling approaches and hence equilibrium droplet diameters are mainly determined by this turbulent quantity, cf. equation [11, 18) and 20). The shift between the modeling of Beau et al. and the one presented here stems from the same fact: Since the approach of Beau et al. features lower collisional velocities than the new one, equilibrium droplet diameters calculated are lower for Beau et al.'s modeling than for the new approach presented here.

Sauter mean diameters obtained by Vallet et al.'s modeling [1], which features a quite different calculation of collisional velocity scale and equilibrium value for $\Sigma$ and $d_{32}$ respectively, lie in between the values obtained with the modeling of Beau et al. and the new approach presented here. Droplet diameters initially decrease to a value of $d_{32} \approx 4 \mu \mathrm{m}$ at $x \approx 12 \mathrm{~mm}$ but then increase to a value of $d_{32} \approx 10 \mu \mathrm{m}$ showing the same trend already described for the other modeling approaches.
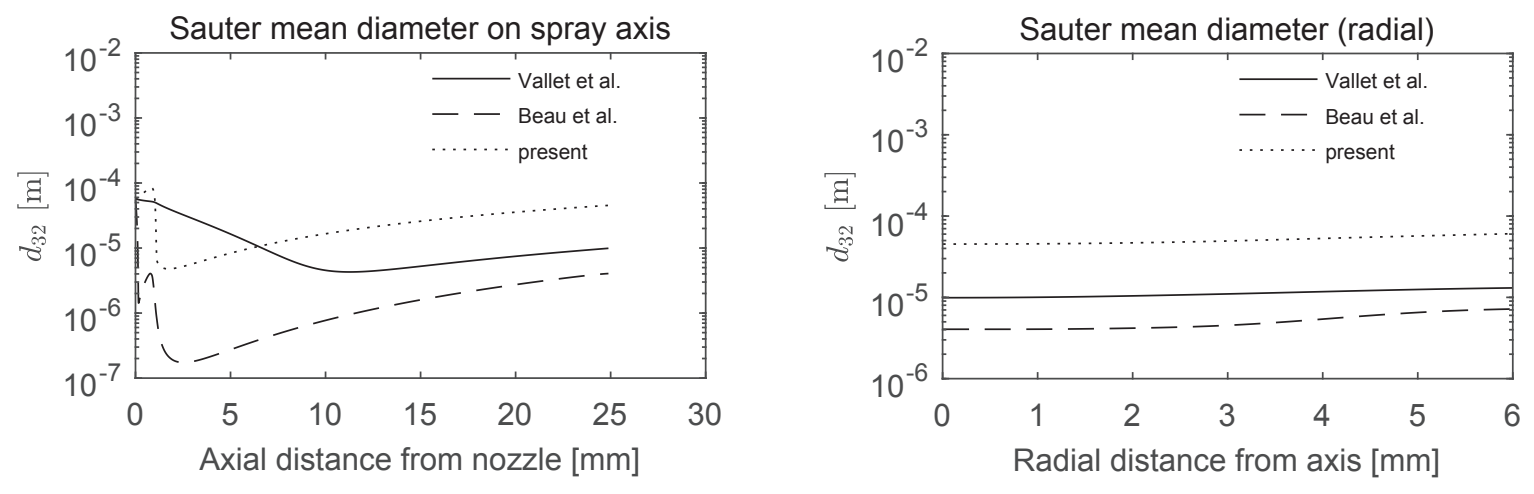

Figure 3. Evolution of Sauter mean diameter along spray axis (left) and radial distribution of Sauter mean diameter at $x=25 \mathrm{~mm}$ (right)

Concerning the radial profile Sauter mean diameters slightly increase when leaving the spray axis. Within Vallet et al's approach this behavior corresponds to decreasing values of turbulent dissipation rate. For the modeling approaches of Beau et al. and the one presented here decreasing values of turbulent kinetic energy are reasonable. The shift in Sauter mean diameters goes along with a shift in turbulent Stokes number, which is presented in Figure 4 The Stokes numbers observed with the new modeling approach are higher than for the modeling approaches of Vallet et al. and Beau et al., cf. Figure 2 in most of the spray. However, Stokes numbers are between St $\approx 0.3 \ldots 0.5$ which still allows the assumption of turbulence induced collisions and hence the application of the present modeling approach to the $\Sigma-Y$ model.

\section{Conclusions}

In the present study, the modeling of collisional velocities applicable to the collisional interfacial area production terms within the $\Sigma-Y$ model was investigated. Two different modeling approaches proposed in the literature were compared against an alternative modeling approach presented here, which includes the influence of Stokes number 


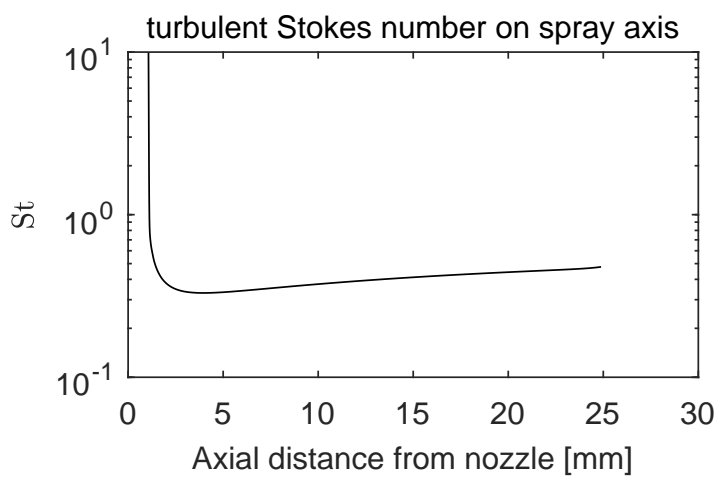

Figure 4. Turbulent Stokes number on the spray axis for the new modeling approach considering Stokes number effects

on the collisional velocity in contrast to the other approaches. It was found that Sauter mean diameters obtained wiith the new modeling approach were larger than the ones calculated if the modeling approaches proposed in the literature were employed.

The modeling approach of Beau et al. and the one presented here are physically similar but differ in the calculation of the actual value for the collisional velocity. The reduced collisional velocities obtained with the new approach (including Stokes number effects) lead to increased values of collisional time scales and equilibrium values for Sauter mean diameters. This again results in increased values for the actual Sauter mean diameters, which are only slightly different from the equilibrium.

Vallet et al. assume a different approach for calculating the collisional velocity and equilibrium Sauter mean diameter. Thus, their approach and the new one presented by the authors was not compared in terms of a superior applicability to the $\Sigma-Y$ model. However, diameters obtained with the new approach roughly have the same order of magnitude as the ones calculated by Vallet et al.'s approach, which has already been successfully applied to spray simulations, see for example [3, 12]. Therefore, an indication of the general applicability of the new modeling for the collisional interfacial area production within the $\Sigma-Y$ model is given.

The new modeling introduced here is supposed to represent an alternative to the modeling approaches of Vallet et al. and Beau et al. But further work is needed to achieve a validation of the modeling proposal, which will be conducted in a continuing study.

\section{Nomenclature}

\section{Roman letters}

$A \quad$ production rate of interfacial area by turbulent stretching $\left[\mathrm{s}^{-1}\right]$

$C \quad$ numerical constant [-]

$d_{32} \quad$ Sauter mean diameter [m]

$k \quad$ turbulent kinetic energy $\left[\mathrm{m}^{2} \mathrm{~s}^{-2}\right]$

$l \quad$ length [m]

$n \quad$ droplet number density $\left[\mathrm{m}^{-3}\right]$

Sc Schmidt number [-]

$T \quad$ time scale [s]

$t \quad$ time [s]

$u \quad$ velocity $\left[\mathrm{m} \mathrm{s}^{-1}\right]$

$Y \quad$ liquid mass fraction [-]

\section{Greek letters}

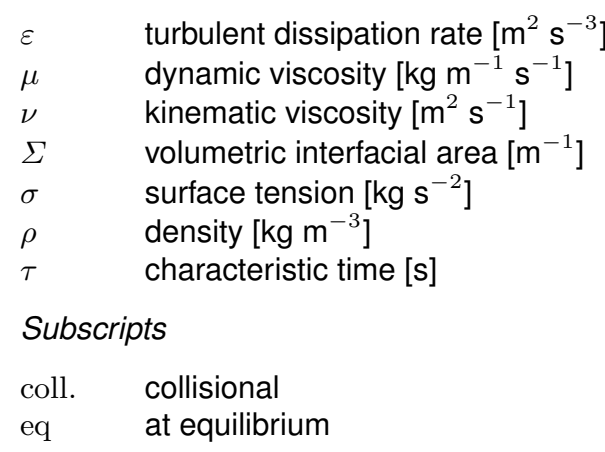




$\begin{array}{ll}\mathrm{f} & \text { fluid } \\ \text { inj } & \text { injection } \\ \text { liq } & \text { liquid } \\ i & \text { in direction } i \\ \text { noz. } & \text { nozzle } \\ \mathrm{P} & \text { production } \\ \mathrm{p} & \text { particle } \\ \mathrm{D} & \text { destruction } \\ t & \text { turbulent }\end{array}$

\section{References}

[1] Vallet A., Burluka, A.A. and Borghi, R., 2001, Development of a Eulerian Model for the "Atomization" of a Liquid Jet, Atomization and Sprays, 11 (6), pp. 619-642.

[2] Lebas, R., Menard, T., Beau, P.A., Berlemont, A., Demoulin, F.X., 2009, Numerical simulation of primary breakup and atomization: DNS and modelling study, Int. J. Multiphase Flow, 35, pp. 247-260.

[3] Garcia-Olíver, J.M., Pastor, J.M., Pandal, A., Trask, N., Baldwin, E. and Schmidt, D.P., 2013, Diesel Spray CFD Simulation based on the $\Sigma-Y$ Eulerian Atomization Model, Atomization and Sprays, 23 (1), pp. 71-95.

[4] Lebas, R., Blokeel, G., Beau, P.-A. and Demoulin, F.X., 2005, Coupling Vaporization Model With the EulerianLagrangian Spray Atomization (ELSA) Model in Diesel Engine Conditions, SAE Technical Paper 2005-01-0213.

[5] Beau, P.A., Menard, T., Lebas, R., Berlemont, A., Tanguy, S. and Demoulin, F.X., July 17.-20. 2006, Numerical Jet Atomization. Part II: Modeling Information and Comparison with DNS Results, FEDSM2006 ASME Joint U.S. - European Fluids Engineering Summer Meeting, Miami, Florida.

[6] Demoulin, F.X., Beau, P.A. Blokeel, G., Arnaud, M. and Borghi, R., 2007, A New Model for Turbulent Flows with Large Density Fluctuations: Application to Liquid Atomization. Atomization and Sprays, 17, pp. 315-345.

[7] Desantes, J.M., Garcia-Olíver, J.M., Pastor, J.M., Pandal, A., Baldwin, E. and Schmidt, D.P., 2016, Coupled/decoupled spray simulation comparison of the ECN spray a condition with the $\Sigma-Y$ Eulerian Atomization Model, Int. J. Multiphase Flow, 80, pp. 89-99.

[8] Pandal, A., Pastor, J.M., Garcia-Olíver, J.M., Baldwin, E. and Schmidt, D.P., 2016, A consistent, scalable model for Eulerian spray modeling, Int. J. Multiphase Flow, 83, pp. 162-171.

[9] Pischke, P. and Kneer, R., Aug. 23.-27. 2015, Droplet Collisions and the Equilibrium Drop Size Distribution in Turbulent Dispersions, 13th Triennial International Conference on Liquid Atomization and Spray Systems, Tainan, Taiwan.

[10] Levins, D.M. and Glastonbury, J.R., 1972, Particle-Liquid Hydrodynamics ans Mass Transfer in a Stirred Vessel Part I Particle-Liquid Motion Trans. Instn. Chem. Engrs., 50, pp. 32-41.

[11] Williams, J.J.E. and Crane, R.I., 1983, Particle Collision Rate in Turbulent Flow, Int. J. Multiphase Flow, 9, pp. 421-435.

[12] Blokeel, G., Barbeau, B. and Borghi, R., 2003, A 3D Eulerian Model to Improve the Primary Breakup of Atomizing Jet, SAE Tech. Pap. 2003-01-0005. 